\section{Belfast, UK}

e-mail:m.gregg@qub.ac.uk

Published online: 18 March 2019

https://doi.org/10.1038/s41563-019-0322-7
References

1. Getzin, S. et al. Proc. Natl Acad. Sci. USA 113, 3551-3556 (2016).

2. Tarnita, C. E. et al. Nature 541, 398-401 (2017).

3. Landau, L. \& Lifshitz, E. Phys. Z. Sowjetunion 8, 153-164

(1935).

4. Kittel, C. Phys. Rev. 70, 965-971 (1946).
5. Stoica, V. A. et al. Nat. Mater. https://doi.org/10.1038/s41563-0190311-x (2019).

6. Yadav, A. K. et al. Nature 530, 198-201 (2016).

7. Haeni, J. H. et al. Nature 430, 758-761 (2004).

8. Mitsui, T. \& Furuichi, J. Phys. Rev. 90, 193-201 (1953).

9. Roytburd, A. L. Phys. Status Solidi A 37, 329-339 (1976).

\title{
Drinking up mist
}

T he harvesting of water droplets from fog on a vertical mesh of fine filaments has been poetically but aptly called 'fishing for water. The droplets coalesce and, held on the threads by capillarity, run downwards under gravity for collection in a trough. The technique has been used at least since the 1960s, especially in mountainous or hilly coastal areas of arid South America and Africa, where fog often appears in the highlands. The droplets are typically collected on a fine mesh of threads made from synthetic polymers such as nylon or polypropylene.

The technology is, at least in principle, cheap and relatively easy to maintain, although water collected this way might sometimes need treatment before being fit to drink, and can be challenging to transport by pipeline in mountainous terrain. The United Nations' World Water Day, which has just passed (22 March), is an annual reminder of how urgently such affordable methods are needed to supplement the overstretched and under-abundant water resources in many areas of the world.

As with any resource harvesting, efficiency is a crucial aspect of economic viability. Every droplet counts. Conventional hydrophilic polymers have the advantage of low cost, but here is an arena where superhydrophilic surfaces can come into its own - not just to capture fog droplets maximally but to minimize water losses as it runs to the collection trough.

One challenge is to combine efficient droplet capture - or in the case of dew rather than fog harvesting, droplet nucleation from vapour - with good liquid transport along the threads. The former is favoured by hydrophilicity, the latter by hydrophobicity to make a 'slippery' surface. However, Dai et al. have shown that hydrophilic surfaces can be made slippery too with some clever nanoscale engineering ${ }^{1}$. They etched silicon wafers with an array of parallel grooves tens of micrometres wide and deep, imparting directional wettability such that water droplets attached to the hydrophilic hydroxylterminated surface are elongated in the direction of the grooves. This texturing of a droplet-collecting surface with microgrooves not only assists transport but can also speed up droplet growth and shedding ${ }^{2}$.

To ensure that the droplets flow smoothly down these 'tracks' under gravity on a tilted surface, the researchers covered the textured surface with hydrophilic liquid lubricants such as hydroxy-terminated polydimethylsiloxanes. Because the lubricant preferentially wets the substrate, it remains in place as the water droplets flow over it. The design was inspired by the microstructures of natural dew- and fog-collecting surfaces such as those on pitcher plant and rice leaves and desert beetles, which also exhibit this combination of hydrophilic surface chemistry, directional textures and slippery surfaces. The engineered surfaces show good fog-collecting performance.

Picking up fog droplets on threads in the first place in moving air is a rather complex

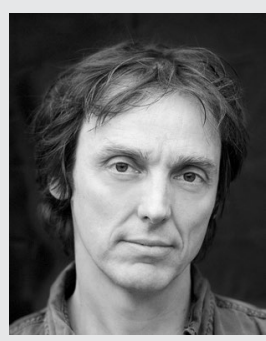

Philip Ball

hydrodynamic process, not least because the drops might be guided around the strands by the airflow instead of colliding with them. And even captured drops might be lost again by evaporation or re-entrainment into the wind - a process that again can be minimized by superhydrophilic materials. Jiang et al. have found that the overall fog collection rate per unit area of fibre surface is proportional to the efficiency of droplet deposition on the fibre ${ }^{3}$. In other words, because aerodynamic effects create higher deposition efficiencies on thinner strands, these also produce a higher water collection rate per unit area of material. That knowledge should assist the optimal design of fog collectors, helping to make the most of their potential to deliver water to where it is needed.

Published online: 20 March 2019 https://doi.org/10.1038/s41563-019-0331-6

References

1. Dai, X. et al. Sci. Adv. 4, eaaq0919 (2018).

2. Bintein, P.-B., Lhuissier, H., Mongruel, A., Royon, L. \& Beysens, D. Phys. Rev. Lett. 122, 098005 (2019).

3. Jiang, Y., Savarirayan, S., Yao, Y. \& Park, K.-C. Appl. Phys. Lett. 114, 083701 (2019). 